Discussion

\title{
Comment on "Equiprobability, Entropy, Gamma Distributions and Other Geometrical Questions in Multi-Agent Systems", Entropy 2009, 11, 959-971
}

\section{Raúl Toral}

IFISC, Instituto de Física Interdisciplinar y Sistemas Complejos, CSIC-UIB, Campus UIB, E-07122 Palma de Mallorca, Spain; E-Mail: raul@ifisc.uib-csic.es; Tel.: +34-971173235

Received: 11 December 2009 / Accepted: 18 December 2009 / Published: 22 December 2009

\begin{abstract}
The volume of the body enclosed by the $n$-dimensional Lamé curve defined by $\sum_{i=1}^{n} x_{i}^{b}=E$ is computed.
\end{abstract}

Keywords: Lamé curves

A recent paper [1] derives asymptotic expressions for the volume of the n-dimensional body defined by $0 \leq \sum_{i=1}^{n} x_{i}^{b} \leq E$ for $b>0, x_{i} \geq 0$. This is the body enclosed by a Lamé curve in $n$ dimensions. Here I compute exactly this volume by using a straightforward modification of the calculation that gives the volume of the n-dimensional sphere, the case $b=2$, see [2].

Writing $E=R^{b}$, the volume $V_{n}(R)$ is

$$
\begin{gathered}
V_{n}(R)=\int_{0 \leq \sum_{i=1}^{n} x_{i}^{b} \leq R^{b}} d x_{1} \cdots \int d x_{n} \\
\text {. }
\end{gathered}
$$

By dimensional analysis $V_{n}(R)=C_{n} R^{n}$. Let us now compute the integral

$$
\int_{0}^{\infty} d x_{1} \cdots \int_{0}^{\infty} d x_{n} \exp \left[-\left(x_{1}^{b}+\cdots+x_{n}^{b}\right)\right]=\left[\int_{0}^{\infty} d x \exp \left[-x^{b}\right]\right]^{n}=\left[\Gamma\left(1+\frac{1}{b}\right)\right]^{n}
$$

by using the change of variables $r=\left(x_{1}^{b}+\cdots+x_{n}^{b}\right)^{1 / b}$ and the volume element $d V_{n}(r)=n C_{n} r^{n-1} d r$ as

$$
\int_{0}^{\infty} d V_{n}(r) \exp \left[-r^{b}\right]=C_{n} \Gamma\left(1+\frac{n}{b}\right)
$$


Equaling these two expressions one gets:

$$
V_{n}(R)=\frac{\left[\Gamma\left(1+\frac{1}{b}\right)\right]^{n}}{\Gamma\left(1+\frac{n}{b}\right)} R^{n}
$$

which is the desired formula. This validates the results in [1], since it coincides with the approximate calculation of that paper in the asymptotic limit $n \rightarrow \infty$ although, as proven here, it turns out to be valid for any value of $n$.

\section{Acknowledgements}

I acknowledge financial support by the MEC (Spain) and FEDER (EU) through project FIS2007-60327.

\section{References}

1. López-Ruiz, R.; Sañudo, J.; Calbet X. Entropy 2009, 11, 959-971.

2. Pathria, R.K. Appendix C. Statistical Mechanics, 2nd ed.; Butterwort-Heinemann: Stoneham, MA, USA, 1996.

(c) 2009 by the author; licensee Molecular Diversity Preservation International, Basel, Switzerland. This article is an open-access article distributed under the terms and conditions of the Creative Commons Attribution license http://creativecommons.org/licenses/by/3.0/. 